\title{
A Mid-Axis extraction of heterogeneous river network data with topological consistency
}

\author{
Hai Hu $1^{\text {a, }}$, Lili Song $2^{\mathrm{a} *}$, Zonglin Yin $3^{\text {a }}$, Min Yang $4^{\mathrm{a}}$ \\ ${ }^{a}$ School of Resources and Environmental Sciences, Wuhan University \\ 1,huhai@whu.edu.cn, \\ 2,songll6_6@163.com \\ 3,hhwhu@163.com \\ 4,YangMin_whu@163.com
}

Keywords: Line surface mixed; River network axis; Mathematical morphology; topology; Map algebra

\begin{abstract}
:
Geographic data in the big data environment presents some new characteristics: diversified data sources, and different ways of obtaining data, which makes the form and content of data different, resulting in diversification of GIS data types. Multiple sources and multiple scales characterize line-surface mixed river data. Different acquisition methods, different acquisition times, and different scales will result in differences in line-surface data and even different resolutions. The river network data used in this experiment usually includes 3 types of river data: surface (Dual-line River), single-line rivers and raster river data from remote sensing images. Among them, the river raster data can be treated as surface data. The surface river data has some specific characteristics. First, the abutment relationship between the line and the surface such as the tributary flow often exists on the main stream. The planar rivers often connect with the linear rivers. Second, the inclusion relationship between the surface and the surface, such as the surface Island phenomenon in the river. Last, the relationship between the two lines and the line, such as the adjoining, intersecting, and separating of the two singleline rivers.

The following methods are used to achieve network topology with line-surface river data. First, the line-surface river data was rasterized into a unified resolution raster so that they have the same data characteristics. Second, the Mid-Axis of the line-surface data is extracted through distance transformation or refine method from mathematical morphology. At last we can establish a topological relationship with the linear data, realize the network topology and apply to network analysis.
\end{abstract}

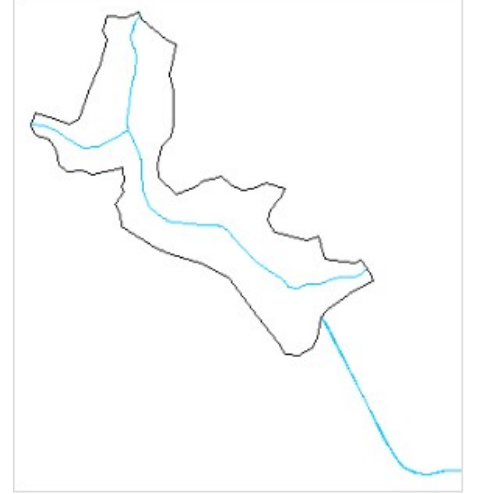

a. morphology-only Axis

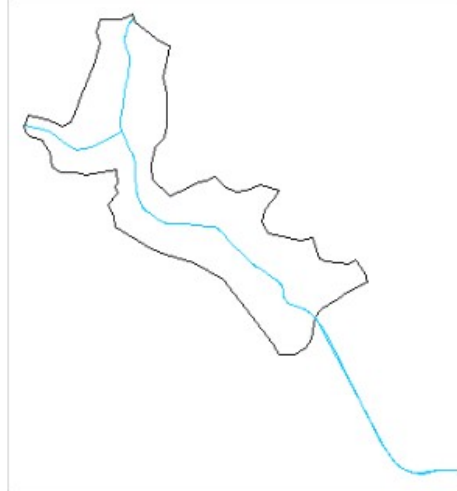

b. Axis with topological consistency

Fig.1 Central axis of Line surface mixed polygon

The blue line in Fig. 1a is based on the linear extraction result of the line-surface mixed data of the Delaunay triangulation, and the line in Fig. 1b is the central axis extraction result of the proposed algorithm. Focus on the connection of the line surface. As can be seen from (a), for the mixed data Delaunay triangulation, only the axis of the planar element can be extracted, and the original single line data cannot be retained. The central axis and the original line data are not established. Topological relationship. The morphological-based refinement method proposed in Figure 1(b) can well avoid the above problems. 

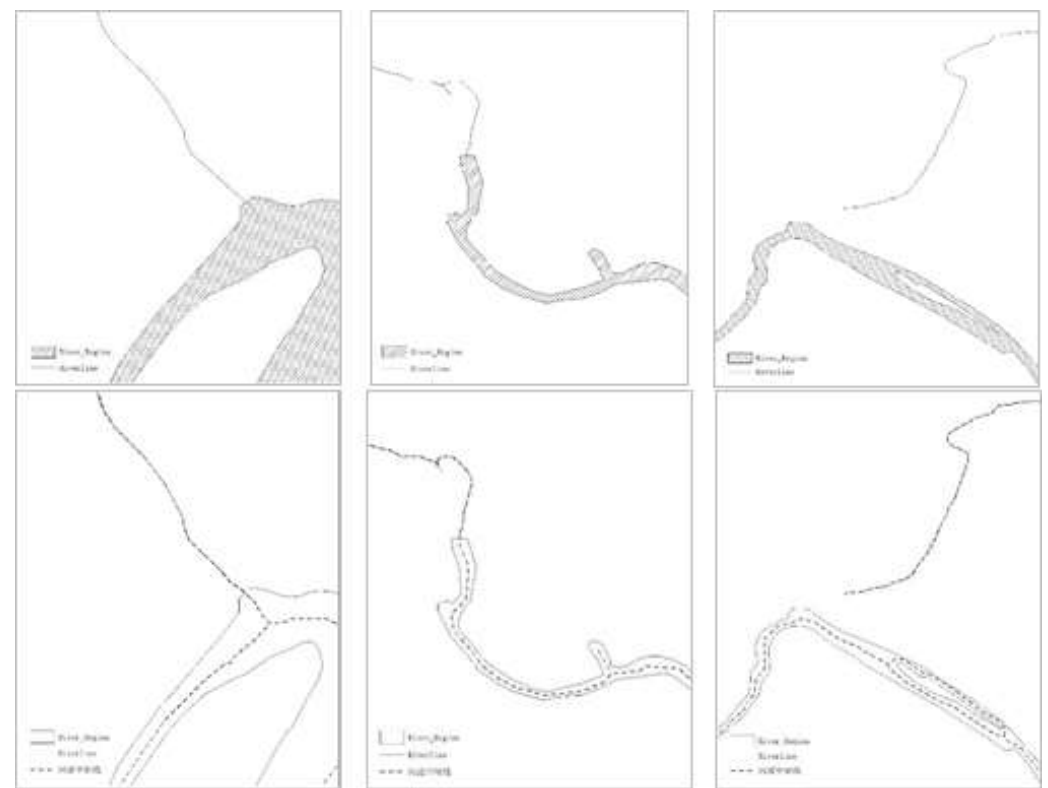

Fig. 2 Central axis extraction results for different line relationships

For the real data experiment, the single-line river that was originally rasterized by linear rivers is well preserved. When a linear river overlaps or is connected to a planar river. The extracted central axis and the single-line river can remain in a completely connected state whenever overlap or junction, and will not adversely affect the extraction result, realizing a fully automated central axis extraction.

The method proposed in this paper is characterized by satisfying the basic requirements of the visualization of the central axis extraction result and achieving full automatic extraction. After the topology check of the extraction result is completed, the network space analysis is directly performed. For example, for the river network axis can be connected for connectivity analysis. Since the data selected in this paper is river network data, the following will establish a network topology and perform connectivity analysis on the results of the central axis extraction.

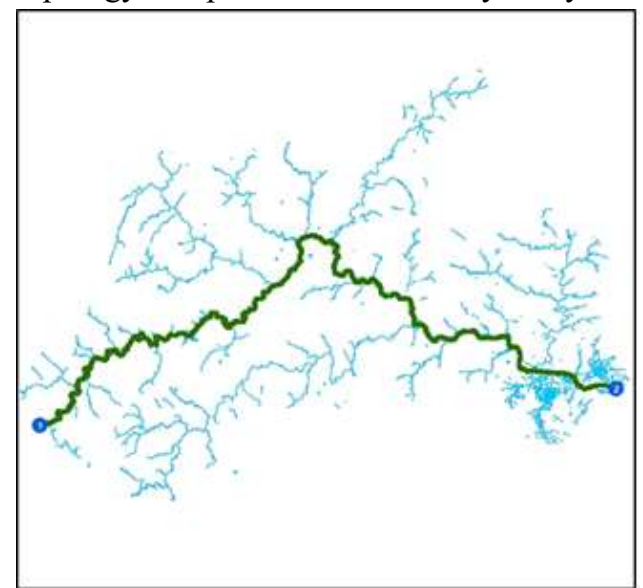

Fig.3 Connectivity Analysis of Central Axis Extraction Results

The two points in Figure 3 are the starting point, point 1 is on the axis of the original linear river, and point 2 is on the central axis of the planar river. The connectivity path is generated between the two points as shown in the figure. The results show that the single-line river network composed of the axes in the line-mixed data extraction has a complete topological connectivity.

River networks are complex spatial data with highly structured features with different scales. The acquisition and establishment of single-line topology river network data is essential for map synthesis and solving the topological relationship of watershed systems, and will play a huge role in GIS applications.

Based on the mathematical morphology and Map Algebra principle, this paper uses the refinement algorithm and template shaping to realize the extraction of the multi-source and multi-scale river central axes of the line-surface mixing, and it is excellent in the transition from the two-line river to the single-line river. The test results show that even for the mixed data of the line surface (including islands) with complex structure, the method can effectively extract the continuous and smooth central axis and maintain a complete topological relationship with the retained single line data. The acquisition and establishment of single-line topology river network data is realized. In the next step of the work, the algorithm will 
be optimized to improve the speed of the algorithm. Although the study in this paper is based on rivers, the research results are equally applicable to other multi-source mixed line data, such as street networks. 